NASA Contractor Report 185265

P.31

\title{
Reliability Analysis of Continuous Fiber Composite Laminates
}

David J. Thomas and Robert C. Wetherhold

State University of New York

Buffalo, New York

July 1990

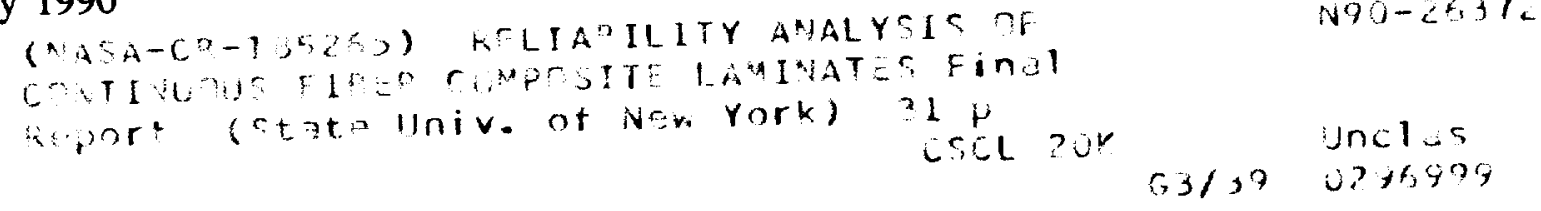

Prepared for

Lewis Research Center

Under Grant NAG3-862

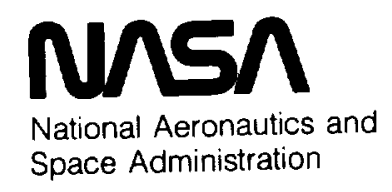


$\ldots$ 


\title{
Reliability Analysis of Continuous Fiber Composite Laminates
}

\author{
David J. Thomas* \\ Robert C. Wetherhold ${ }^{\dagger}$ \\ Department of Mechanical and Aerospace Engineering \\ State University of New York \\ Buffalo, New York 14260
}

\begin{abstract}
A composite lamina may be viewed as a homogeneous solid whose directional strengths are random variables. Calculation of the lamina reliability under a multi-axial stress state can be approached by either assuming that the strengths act separately (modal or independent action), or that they interact through a quadratic interaction criterion. The independent action reliability may be calculated in closed form, while interactive criteria require simulations; there is currently insuffcient data to make a final determination of preference between them. Using independent action for illustration purposes, the lamina reliability may be plotted in either stress space or in a non-dimensional representation. For the typical laminated plate structure, the individual lamina reliabilities may be combined in order to produce formal upper and lower bounds of reliability for the laminate, similar in nature to the bounds on properties produced from variational elastic methods. These bounds are illustrated for a $[0 / \pm 15]$, Graphite/Epoxy(GR/EP) laminate. In addition, simple physically plausible phenomenological rules are proposed for redistribution of load after a lamina has failed. These rules are illustrated by application to $[0 / \pm 15]$, and $[90 / \pm 45 / 0]$, GR/EP laminates and results are compared with respect to the proposed bounds.
\end{abstract}

* Graduate Student

${ }^{\dagger}$ Associate Professor 


\section{Introduction}

The role of composite materials in advanced engineering applications has created a need for improved failure analysis capabilities. The major existing failure theories in present use (Maximum Stress, Tsaj-Wu, Tsai-Hill, Maximum Distortional Energy, etc.) are deterministic models borrowed or adapted from use with conventional homogeneous isotropic materials. However, composite materials inherently possess a high variability in material strengths. This is due in part to the heterogeneous make-up of composites, as well as from effects of the manufacturing process. In order to utilize composites most effectively, their failure analysis must be approached from a probabilistic standpoint. This will allow us to meet desired reliability goals, while avoiding costly overdesign. Before engaging in a probabilistic description of fracture, however, we must consider the exact mechanics of failure which we will model for a composite laminate.

Composite laminates under in-plane loadings typically fail through a combination of the following modes: matrix normal stress, matrix shear, delamination, and fiber failure. These failure modes have been the focus of much research in recent years, and the results of these efforts have proven useful in understanding the mechanisms behind the failure modes. Many of the published articles deal with the micro-mechanic(fiber and matrix) level, especially for fiber failure. Unfortunately, the complex nature of the failure process has hindered the development of a failure analysis scheme which would be capable of considering modal interactions over an element of practical volume, let alone the interaction between laminae of finite volume. In this paper a macroscopic approach to failure has been been chosen, thus allowing the development of phenomenological models to analytically describe the reliability limits of the composite as well as the load redistributions occurring within the laminate as internal failures develop.

Of the four modes of failure mentioned, matrix normal and shear cracking

and fiber failure come as a result of in-plane stresses. Both categories of delamination, namely free edge delaminations and localized delaminations which occur internally at matrix crack tips, are due to the development of interlaminar stresses[1,2]. Careful design of the lay-up can help reduce or eliminate the stresses which cause delamination. It will be assumed that this is the case here, and that the failure of the laminates under study will not be dominated by delaminations. This enables the failure function to be considered as a function of only in-plane stresses.

In applying reliability methods to composite laminates, we will first con- 
sider lamina failure probabilities, then propose formal limits for laminate reliability. These limits are reliability bounds similar in nature to the bounds for elastic properties. By proposing sensible load sharing rules for failed laminae within a laminate, we will assess the importance of load-sharing on laminate reliability. Recent publications considering reliability analysis of laminates have proceeded in a similar direction, but with somewhat less general results. Yang[13] utilized an interactive criterion only, did not account for localized effects during load redistribution, and relied on the use of a failure tree(the drawbacks of which are discussed in Section(4) of this paper). Fukunaga et al.[14] accounted for localized effects through the use of strain concentration factors, but they too relied on an interactive criterion and the use of failure trees. Additionally, the strength parameters used in this model are laminate specific.

\section{Reliability Analysis of a Single Layer}

The reliability analysis used in this paper for the uniaxial strengths of a lamina are based upon a Weibull weak link formulation. For the present research, the volume of interest will be taken to be the volume of an individual lamina. The lamina is assumed to be statistically homogeneous in its elastic and strength properties, thus making the Weibull parameters constant over the volume of the lamina. The layer's strengths, $X_{i}$, are assumed to each have a Weibull distribution of the form,

$$
F_{X_{i}}\left(\sigma_{i}\right)=1-\exp \left[-\psi_{i}\right]
$$

where

$$
\psi_{i}=\left(\frac{\sigma_{i}}{\beta_{i}}\right)^{\alpha_{i}} \quad i=1,2,6
$$

The Weibull parameters $\left(\alpha_{i}, \beta_{i}\right)$ describing the strength distribution for the material are determined experimentally from uniaxial tests[3,4]. If the volume from which $\beta_{i}$ was experimentally based is not the same as the lamina volume to which the analysis is being applied, the following volume correction to $\beta_{i}$ is applied.

$$
\beta_{i}=\left(\frac{V^{\prime}}{V}\right)^{1 / \alpha_{i}} \beta_{i}^{\prime}
$$

where:

$\beta_{i}^{\prime} \quad$ is the value of the scale parameter for the test specimen 
$\beta_{i} \quad$ is the desired scale parameter for the lamina

$V^{\prime}$ is the volume of the test specimen

$V \quad$ is the volume of the lamina

The stresses $\sigma_{1}, \sigma_{2}$, and $\sigma_{6}$ have the usual meaning of longitudinal, transverse and shear stresses in the lamina material coordinates. While it is clear that the probability of failure per unit volume as given in Equation(2) may be used for uniaxial cases, it is not sufficient to describe cases where the lamina is under a state of plane stress. For such instances, a multiaxial failure density function, $\psi\left(\sigma_{1}, \sigma_{2}, \sigma_{6}\right)$, must be formulated. Both interactive and non-interactive forms for the failure function will be investigated.

Non-interactive Failure Criterion:In a non-interactive failure criterion, the stresses present on an element are assumed to act independently of one another towards the failure of that element, thus lending the name Principle of Independent Action(PIA) to this analysis. Utilizing the two parameter Weibull distribution for strength that was given in Equation(2) yields the following form for the reliability of a single layer,

$$
R=\exp \left[-\sum_{i=1,2,6} \psi_{i}\right]
$$

and where $\psi_{i}$ is further defined in more general terms as below to allow consideration of both tensile and compressive stress values.

$$
\begin{gathered}
\psi_{i}=\left(\frac{\left\langle\sigma_{i}\right\rangle}{\beta_{i}^{T}}\right)^{\alpha_{i}^{T}}+\left(\frac{\left\langle-\sigma_{i}\right\rangle}{\beta_{i}^{C}}\right)^{\alpha_{i}^{C}} \\
\langle x\rangle \equiv x \cdot u[x]= \begin{cases}0, & \text { if } x \leq 0 \\
x, & \text { if } x>0 .\end{cases} \\
\alpha_{i}^{T}, \beta_{i}^{T}: \text { Weibull parameters for tension } \\
\alpha_{i}^{C}, \beta_{i}^{C}: \text { Weibull parameters for compression }
\end{gathered}
$$

Interactive Failure Criterion: An interactive approach considers the stresses to work collectively towards the failure of the element, which certainly seems more physically plausible than PIA. The disadantage of such a law is that it only allows a fail/no fail determination for the lamina. No additional information(i.e. key stresses, mode of fracture, etc.) can be extracted. To the knowledge of the authors, a satisfactory model for an interactive failure 
function per unit volume is not available in closed form and must therefore be considered using simulation techniques. This approach is taken with the understanding that should a suitable model for $\psi$ be found, its implementation can be made in a straight forward manner.

Many of the typical failure functions(Tsai-Wu, Maximum Distortional Energy, etc.) have been investigated and found to produce similar results [5]. For purposes of illustration, the Maximum Distortional Energy failure function, MDE, has been chosen as the foundation for the interactive reliability analysis in this paper. It is given in its deterministic form in Equation(6),

$$
f=\left(\frac{\sigma_{1}}{X_{1}}\right)^{2}+\left(\frac{\sigma_{2}}{X_{2}}\right)^{2}-\frac{\sigma_{1} \sigma_{2}}{X_{1}^{2}}+\left(\frac{\sigma_{6}}{X_{6}}\right)^{2}
$$

where the $\sigma_{i}$ are the known applied stresses, and the $X_{i}$ are the deterministic strength values. In its deterministic form, $f<1$ denotes survival, and $f>1$ denotes failure; from this, no probabilistic inference can be made. If, however, the strength values used in Equation(6) are considered as random variables, then the reliability of a layer under a specified load can be stated as the probability that the value of $f$ is less than one for that load.

$$
R_{\text {layer }}=\operatorname{Pr}(f<1)
$$

The integrations necessary for a direct calculation of the reliability are intractable. For this reason, a Monte Carlo computer simulation is normally used for the analysis $[6,7,9,11]$. Through inversion of the probability distribution function for the Weibull random variable $X_{i}$, a set of realizations for $X_{i}$ is obtained. Applying the failure function given in Equation(6) to the simulated sample population allows for a statistical determination of the reliability [i.e. $\operatorname{Pr}(f<1)$ ] for a given stress state. If the volume of the test specimen is different from the volume of the lamina under consideration, there is only one way to apply a volume correction consistent with weak link modelling, mainly this is to scale the reliability itself using the known volume of the test specimen, and not to scale the individual strengths independently.

$$
R=\left(R^{\prime}\right)^{V / V^{\prime}}
$$

where:
$R^{\prime} \quad$ is the value of the reliability for the test specimen
$R \quad$ is the desired reliabilty for the lamina
$V^{\prime} \quad$ is the volume of the test specimen
$V \quad$ is the volume of the lamina 


\begin{tabular}{|ccccc|}
\hline \multicolumn{4}{|c|}{ Weibull Strength Parameters } \\
\hline$X_{i}$ & $\alpha^{T}$ & $\alpha^{C}$ & $\beta^{T}=\beta^{C}$ \\
& & & $M P a$ & $\left(10^{3} p s i\right)$ \\
\hline$X_{1}$ & 25. & 37.5 & 1516. & 220. \\
$X_{2}$ & 10. & 15. & 51.7 & 7.5 \\
$X_{6}$ & 15. & 22.5 & 68.9 & 10. \\
\hline
\end{tabular}

Table 1: Weibull strength parameters for Graphite/Epoxy [3]

Reliability Surfaces: Failure surfaces plotted in stress space are a common design aid for conventional materials as well as for composite materials. This concept can be expanded to the idea of plotting surfaces of constant reliability[5,8]. A range of reliability surfaces using a PIA analysis for a $\mathrm{Gr} / \mathrm{Ep}$ lamina, are shown in Figure(1). Model strength parameters for Gr/Ep are given in Table(1).

It is possible to standardize these surfaces by mapping them into a nondimensionalized space. Introducing a new term of the form:

$$
\xi_{i}^{2} \equiv \psi_{i} \quad i=1,2,6
$$

the failure density function, $\psi$, becomes,

$$
\psi=\xi_{1}^{2}+\xi_{2}^{2}+\xi_{6}^{2}
$$

Equation(10) provides a direct octant to octant mapping of the surface from a stress space to a reliability oriented space where the surface becomes spherical nature. Non-dimensionalized surfaces for the same Gr/Ep material are illustrated in Figure(2). Note that in $\xi$-space the reliability surfaces will be material independent; this results in a series of concentric reliability surfaces which are spherically symmetric about the coordinate axes. This is not necessarily the case in a stress space, as has already been demonstrated by Figure(1).

\section{Bounds on the Reliability of a Laminate}

In expanding the analysis from a lamina to a laminate, formal bounds for the reliability limits of a laminate may be determined. First however, the unit of failure to be considered must be defined. Two such units are proposed here: 


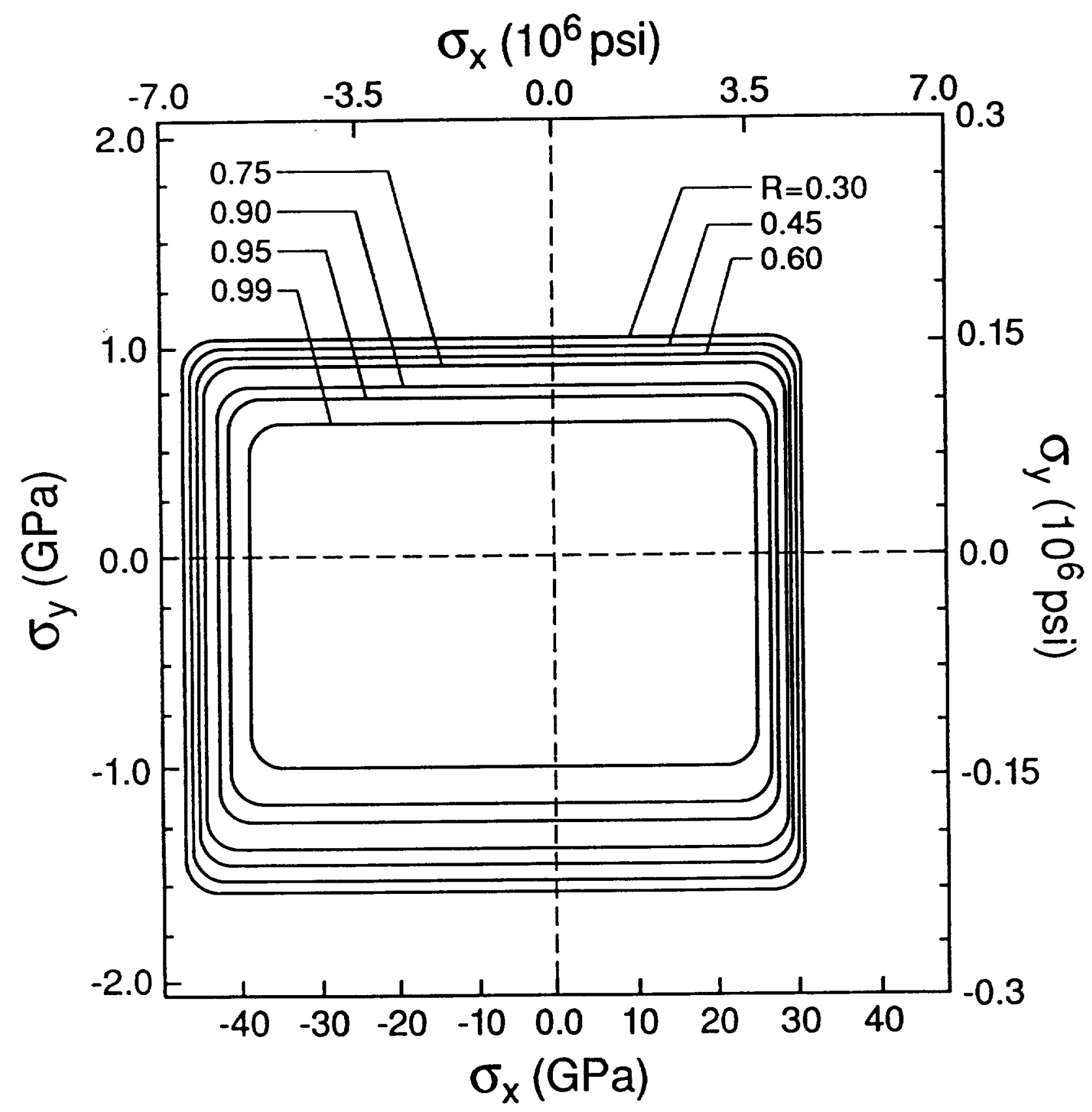

Figure 1: Reliability surfaces for Graphite/Epoxy lamina in stress space 


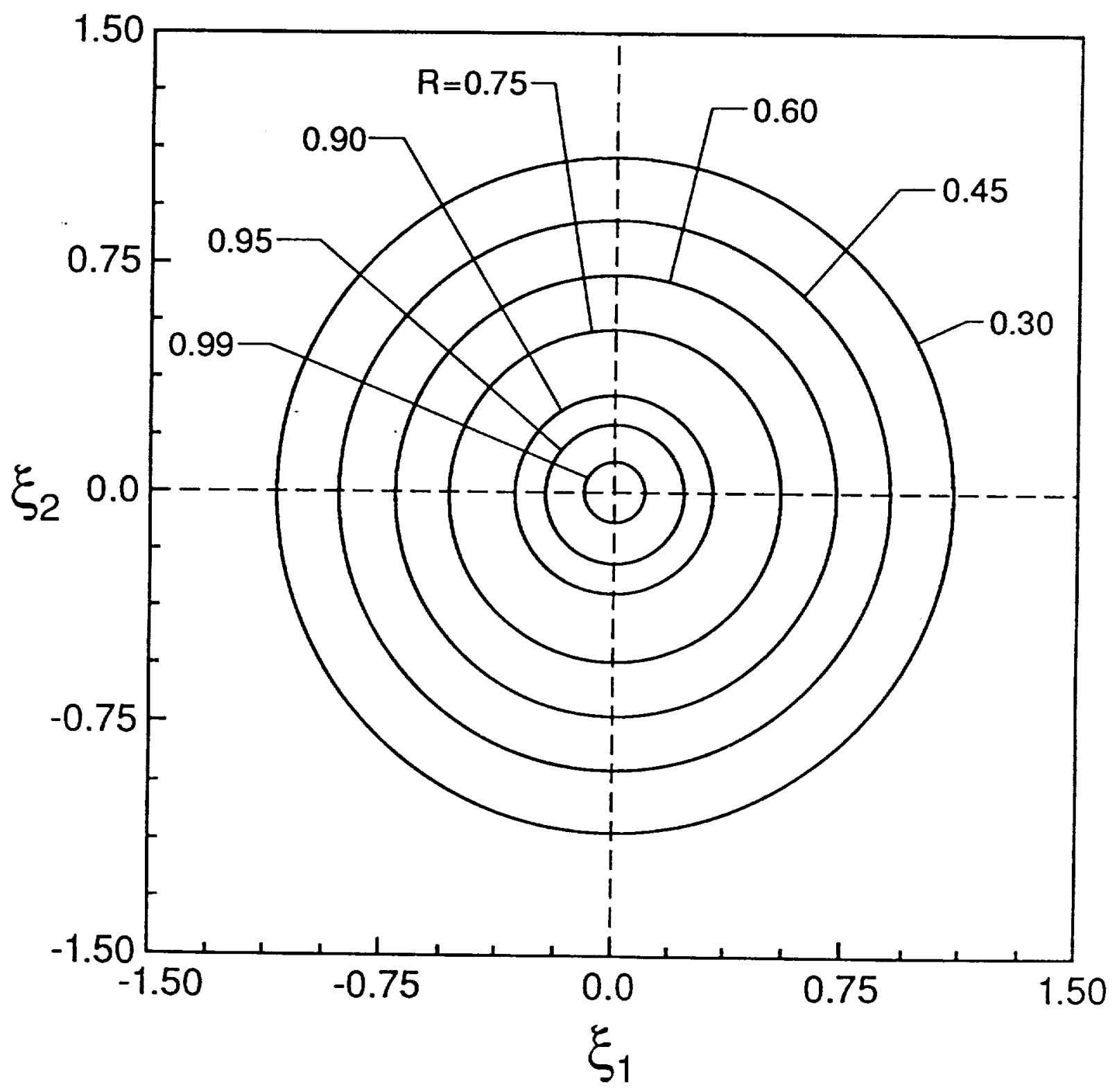

Figure 2: Generalized reliability surfaces in non-dimensionalized space 
1. Ply failure considerations assume that the units of failure are the individual plies which make up the laminate; thus in an $n$-ply laminate there are $n$ failure units. This assumption is implicit in using an interactive criterion.

2. Modal failure considerations allow for the recognition of three potential modes of failure(longitudinal, transverse and shear) within each of these plies, thereby resulting in $3 n$ failure units for an $n$-ply laminate. This assumption is naturally attuned to the use of PIA criteria.

Establishing the Reliability Limits: A lower bound limit for the reliability may be found by applying a weakest link criterion to the laminate. This can take the form of either a first ply or a first mode failure theory, and in essence neglects all in-situ responses such as constraint effects and reloading of damaged plies. The individual failure units are assumed to behave independently, therefore the lower bound laminate reliability is given as the product of the individual unit reliabilities. For the case of the ply failure consideration, this results in

$$
R_{L_{p l y}}=\prod_{i=1}^{n} R_{i}
$$

where $R_{i}$ are the ply reliabilities computed using either a PLA or MDE analysis. For the modal failure considerations, the resulting expression is

$$
R_{L_{\text {modal }}}=\prod_{i=1}^{n} \prod_{j=1,2,6} R_{i j}
$$

where $R_{i j}$ is the reliability of the $j^{\text {th }}$ mode of layer $i$ and is computed by appropriately partitioning Equation(4).

An upper bound reliability limit determination can be made by taking an opposite approach, that is to say by assuming a strongest link theory. Such a statement assumes the influence of the laminate upon the individual failure elements to dominate, so that ultimate failure of the laminate will not occur until every individual unit has failed. Thus the probability of failure for the laminate is given by the product of the probabilities of failure for the individual units. In terms of reliabilities this gives the following expressions for the upper bound limits: for ply failure considerations,

$$
R_{U_{p l y}}=1-\prod_{i=1}^{n}\left(1-R_{i}\right)
$$




\begin{tabular}{|cccc|}
\hline \multicolumn{4}{|c|}{ Weibull Strength Parameters } \\
\hline$X_{i}$ & $\alpha^{T}=\alpha^{C}$ & \multicolumn{3}{c|}{$\beta^{T}=\beta^{C}$} \\
& & $M P a$ & $\left(10^{3} p s i\right)$ \\
\hline$X_{1}$ & 25. & 1516. & 220. \\
$X_{2}$ & 10. & 51.7 & 7.5 \\
$X_{6}$ & 15. & 68.9 & 10. \\
\hline
\end{tabular}

Table 2: Weibull strength parameters for Graphite/Epoxy [3]

and for modal failure considerations,

$$
R_{U_{\text {modal }}}=1-\prod_{i=1}^{n} \prod_{j=1,2,6}\left(1-R_{i j}\right)
$$

Note that in this formulation, if a particular mode is not loaded, and thus has a modal reliability value of $R_{i j}=1.0$, the product term in Equation(14) will become exactly zero thereby returning a value of unity for $R_{U_{\text {modal }}}$. In order to compensate for this occurrence, only modes which are actively loaded during the life of the laminate should be included in Equation(14). As an example, consider a cross-ply laminate under uniaxial loading. Regardless of the magnitude reached by the loading, the shear stresses within the plies will remain at zero, and therefore the $R_{i 6}$ terms should be omitted from the calculation.

The reliability limits have been applied to a $[0 / \pm 15]_{s}$ GR/EP laminate having layer thicknesses of $t=0.005 \mathrm{in}$. and uniaxial loading conditions present(i.e. $N=\left[\begin{array}{lll}N_{x} & 0 & 0\end{array}\right]^{T}$ ). To clarify the results, the shape parameters for tension and compression have been set equal, see Table(2). The elastic material properties for GR/EP are given in Table(3). In Figure(3) the results of the limit analysis have been shown in the form of a plot of reliability versus applied load. Note that the interactive or ply failure curves show lower failure loads than the modal failure curves; this is an indication of the dominance of one of the strength terms. In ply failure, this one strength causes failure of the lamina; while in modal failure, this effect is diluted since all strengths are considered.

Reliability Surfaces: The idea of a surface of constant reliability from may be extended to encompass upper and lower bound reliability limits for a composite laminate. Upon defining the laminate(i.e. layer orientations and layup), the loading $\operatorname{ratios}\left(N_{x}: N_{y}, N_{x}: N_{x y}\right)$ and the desired reliability value, an iteration scheme may be employed to solve for the loading mag- 


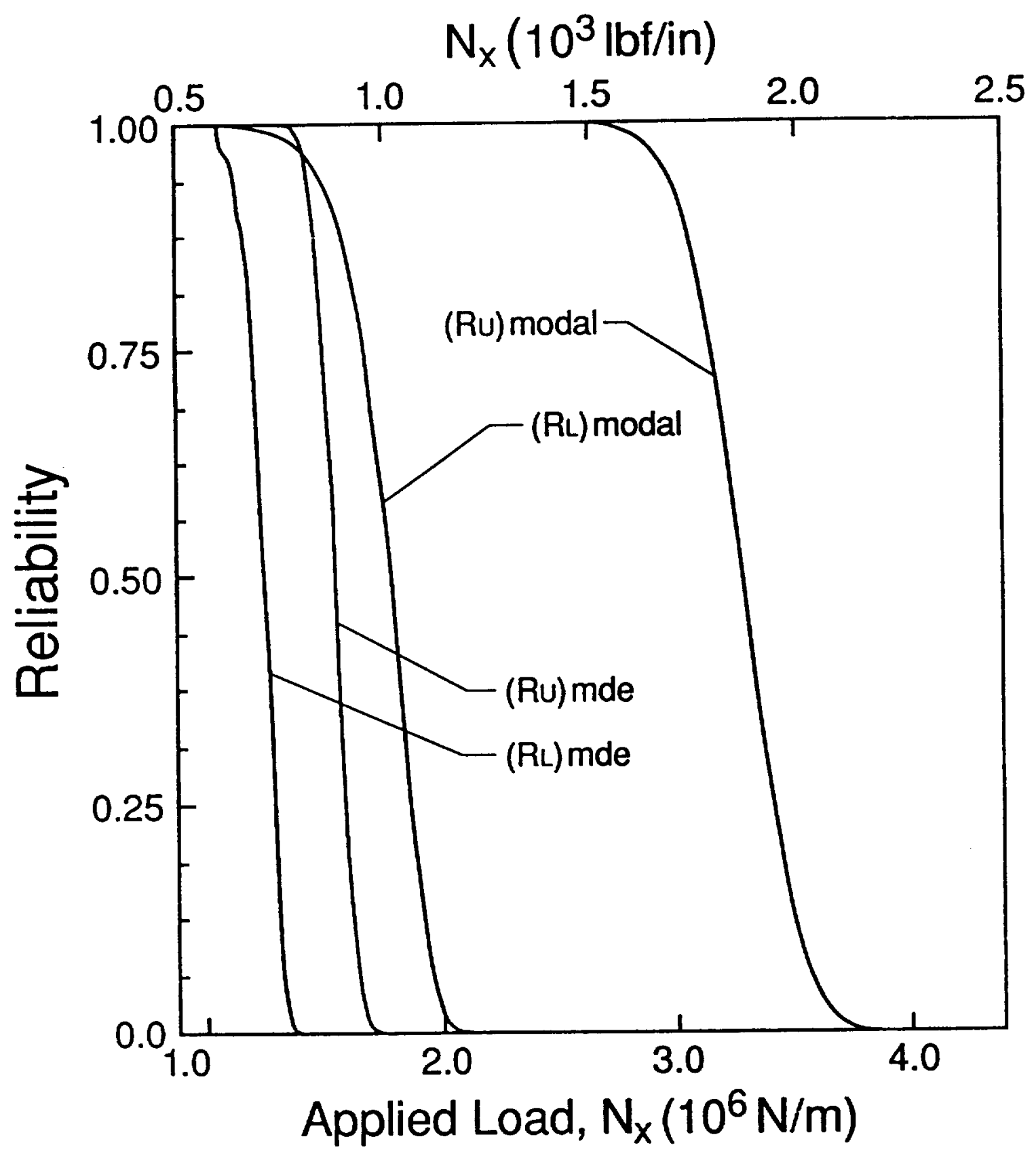

Figure 3: Reliability limit analysis for a $[0 / \pm 15]$, GR/EP using both ply and modal failure criterion 


\begin{tabular}{|lll|}
\hline \multicolumn{3}{|c|}{ Elastic Properties } \\
\hline$E_{1}$ & $125 . \times 10^{3} \mathrm{MPa}$ & $\left(18.2 \times 10^{6} \mathrm{psi}\right)$ \\
$E_{2}$ & $11.2 \times 10^{3} \mathrm{MPa}$ & $\left(1.63 \times 10^{6} \mathrm{psi}\right)$ \\
$G_{12}$ & $7.38 \times 10^{3} \mathrm{MPa}$ & $\left(1.07 \times 10^{6} \mathrm{psi}\right)$ \\
$\nu_{12}$ & 0.236 & \\
\hline
\end{tabular}

Table 3: Elastic material properties for Graphite/Epoxy [3]

nitudes where the upper and lower reliability limits have the desired value. A computer program may be written to systematically determine the necessary data points to generate surfaces of constant reliability for the laminate in a load $\left(N_{x}, N_{y}, N_{x y}\right)$ space. This can be done for both PIA and MDE analyses. Limit surfaces for a constant 90 percent reliabilty are shown in Figure(4) for a $[0 / \pm 15]_{\text {s }} \mathrm{Gr} / \mathrm{Ep}$ laminate under a state of biaxial loading $\left(\underline{N}=\left[\begin{array}{lll}N_{x} & N_{y} & 0\end{array}\right]^{T}\right)$.

\section{Load Redistribution Considerations}

The failure models presented thus far for laminate analysis have only been able to provide bounds of reliability for a given problem. These bounds are often too far apart to be of practical use; therefore it becomes essential to be able to determine a more precise reliability value. The next step in trying to achieve a better reliability analysis is to more closely model the physics of the failure process. Note that the key phrase here is more closely and not exactly. This is because in order to exactly model the process, the development and interaction of cracks on the microscopic scale would have to be considered. Attempting to consider all the possible failure sequences at this level would be impossible. However, by moving to a macroscopic level and making certain simplifying assumptions, a phenomenological model can be reached which is both tractable and emulates the actual failure sequence.

\subsection{Reliability Determination Using Computer Simulations}

In this section two different failure algorithms, corresponding to interactive and non-interactive criteria will be presented along with various load redistribution schemes. First, however, the process to be modeled should be stated in a more detailed manner. The laminate is assumed to be loaded multi-axially, from zero to the designated load in the manner of a load controlled test. As the loading is applied, various failure mechanisms (such as 


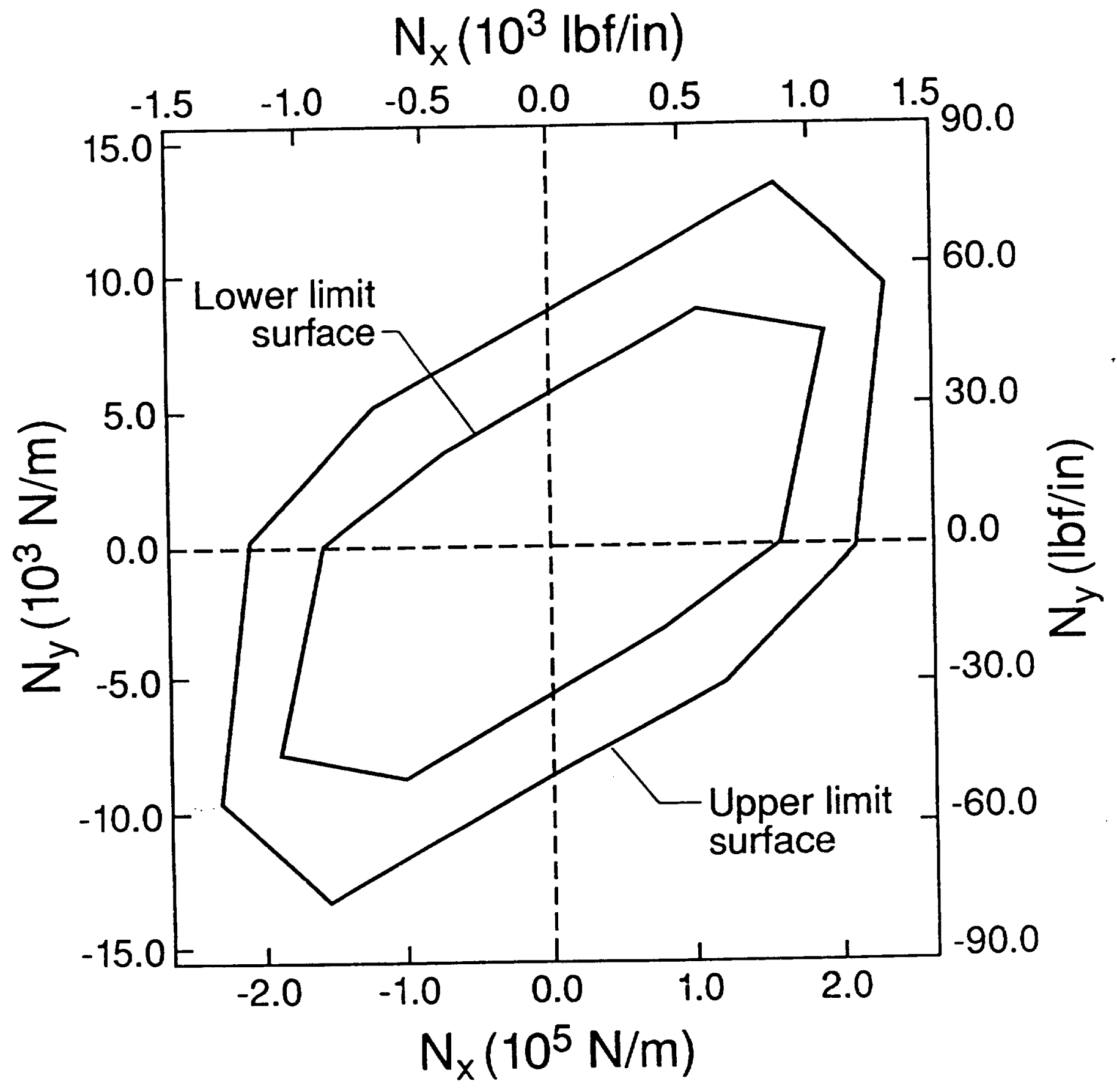

Figure 4: Limit surfaces of constant 90 percent reliability for $[0 / \doteq 15]$ s GR/EP using a PIA analysis 


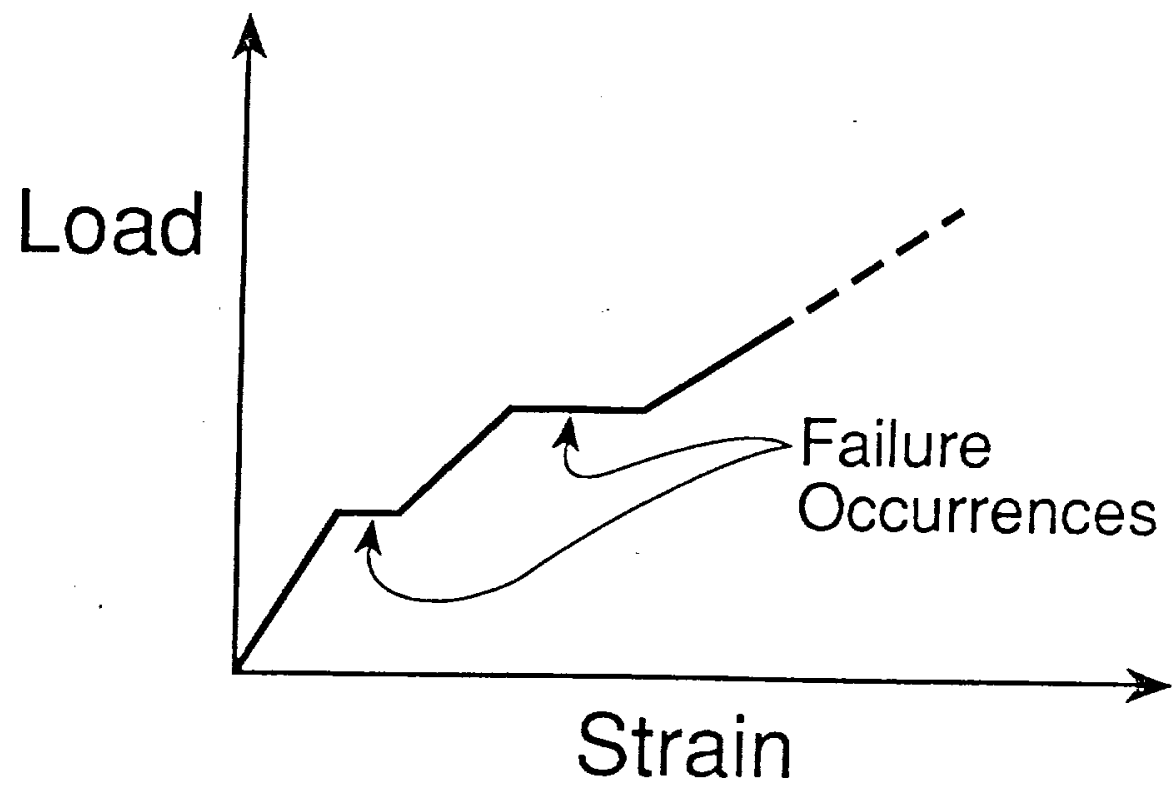

Figure 5: Load - Displacement diagram

those discussed in the introduction) are induced within the individual layers. These failures result in stiffness degradations and load redistributions. The general form of the load-displacement diagram would appear as in Figure(5).

Thus the problems which must be addressed are:

- Defining what constitutes a failure and when it occurs.

- Detailing the method of load redistribution from the failed element.

- Modelling the corresponding stiffness degradations induced by the failures.

The ideal modeling of the failure process would create a failure tree with all possible fracture sequences and their redistributions. This failure tree suffers from two problems: the number of branches and their at tendant failure rules quickly becomes very large. and the addition of laminae requires the entire tree to be rebuilt. A more appropriate method then. is to simulate strengths in the manner of Section(2) and to arrive at a set of realizations for laminate strengths according to the rules we select. In this fashion. the laminate reliability can be calculated, and the load sharing rules easily 
changed. In a simplification for clarity, we shall neglect the effect of bendingextensional coupling, i.e. symmetry of lamina strengths is assumed.

Simulation With an Interactive Failure Criterion: For an interactive failure criterion, the failure element is an individual ply. Defining failure in this way has the adantage of satisfying an intuitive feeling that all stress components should contribute in some collective fashion towards the failure of the layer. However, it has the disadvantage of giving no information as to the mode by which failure occurred; namely longitudinally, transversely or by shear. The lack of such information requires the assumption of uniform reductions in the load carried by the layer(i.e. $\sigma_{1}, \sigma_{2}$ and $\sigma_{6}$ are all reduced by the same factor) and in the layer's stiffness matrix (i.e. all the elements of layer stiffness matrix must also be reduced by this same factor). As a conservative estimate, it will be assumed that the failed layer completely unloads and the stiffness matrix becomes a zero matrix. All that remains is to determine the manner in which to redistribute the load which had been carried by the now failed element. Several physically plausible possibilities exist, and are explained below.

Global Redistribution: The load is redistributed among all remaining unfailed plies in accordance with the laminate constitutive law which accounts for the relative stiffnesses of the layers(including the failed layers in which the appropriate stiffness elements have been set equal to zero) and with the deformation assumptions requiring plane sections to remain planar and perpendicular to the mid-surface.

Local Redistribution, Orientation Insensitive: The load is evenly distributed between the two adjacent layers. If either of these plies has already failed, that portion of the load is globally redistributed.

Local Redistribution, Orientation Sensitive: The load is distributed between the two adjacent layers, with the fraction of the load going to each layer being a function of the relative angles between the respective layers and the failed layer. This uses the physical intuition that fibers in one layer tend to reinforce the matrix direction of neighboring layers if the layers are perpendicular. The functional dependence should result in the layer with the smallest relative orientation accepting the smaller share of the redistributed load. The following relationship has been chosen to determine the percentage of the load from failed layer $i$ to be distributed to the neighboring lavers $i-1$ and $i+1$.

$$
r_{i-1}+r_{i+1}=1
$$




$$
r_{i-1} \equiv \begin{cases}\frac{\cos ^{2}\left(\Delta \theta_{i-1}\right)}{\cos ^{2}\left(\Delta \theta_{i+1}\right)} r_{2}, & \text { if } \Delta \theta_{i+1} \neq 90^{\circ} \\ 1, & \text { if } \Delta \theta_{i+1}=90^{\circ}\end{cases}
$$

where the $r$ 's and $\Delta \theta$ 's represent the load percentages and the relative angles, respectively. As in the orientation sensitive case, any load which cannot be locally redistributed due to previous failures in the neighboring plies will be globally distributed.

Tapered Redistribution, Orientation Insensitive: The load is distributed among the four neighboring layers. The two immediately adjacent layers receive one third of the total load each, and the two outside layers each accept one sixth of the loading. Here again, any load which cannot be distributed locally is done so globally.

Tapered Redistribution, Orientation Sensitive: The load is redistributed among the four neighboring plies with a functional dependence existing between the fractional distribution of the load and the relative angles between the respective layers and the failed layer. The redistribution scheme is presented below, using similar notation to above:

$$
\begin{aligned}
& r_{i-1}+r_{i+1}=\frac{2}{3} \\
& r_{i-2}+r_{i+2}=\frac{1}{3} \\
& r_{i-1} \equiv \begin{cases}\frac{\cos ^{2}\left(\Delta \theta_{i-1}\right)}{\cos ^{2}\left(\Delta \theta_{i+1}\right)} r_{i+1}, & \text { if } \Delta \theta_{i+1} \neq 90^{\circ} \\
1, & \text { if } \Delta \theta_{i+1}=90^{\circ}\end{cases} \\
& r_{i-2} \equiv \begin{cases}\frac{\cos ^{2}\left(\Delta \theta_{i-2}\right)}{\cos ^{2}\left(\Delta \theta_{i+2}\right)} r_{i+2}, & \text { if } \Delta \theta_{i+2} \neq 90^{\circ} \\
1, & \text { if } \Delta \theta_{i+2}=90^{\circ}\end{cases}
\end{aligned}
$$

Once again, any load remaining after local redistribution is redistributed globally. A more detailed discussion of the loading, redistribution and reliability determination is given in Appendix A.

Simulation With a Non-interactive Modal Failure Criterion:

With a non-interactive modal failure criterion, there are three potential modes of failure in each layer - longitudinal, transverse and shear. With the failure function for each mode being given by the absolute value of the ratio of the appropriate stress to the corresponding strength.

Longitudinal:

$$
f_{1}=\left|\frac{\sigma_{1}}{X_{1}}\right|
$$


Transverse:

$$
f_{2}=\left|\frac{\sigma_{2}}{X_{2}}\right|
$$

Shear:

$$
f_{6}=\left|\frac{\sigma_{6}}{X_{6}}\right|
$$

Failure in each case is defined by:

$$
\begin{array}{cc}
f_{i}<1, & i=1,2,6 \quad \text { survival of mode } \\
f_{i} \geq 1, \quad i=1,2,6 & \text { failure of mode }
\end{array}
$$

As before, the strengths, $X_{1}, X_{2}$ and $X_{6}$, are taken to be random variables with a Weibull distribution.

The advantage of modal definitions of failure is the ability to implement a more detailed unloading scheme. For example, if a layer experiences a longitudinal failure the load carried in that direction, $\sigma_{1}$, and the associated stiffness elements, $Q_{11}$ and $Q_{12}$, are reduced and the other loading and stiffness terms remain unchanged. Similarly, for a transverse failure the stress $\sigma_{2}$, and the $Q_{22}$ and $Q_{12}$ stiffness terms are reduced; and for a shear failure $\sigma_{6}$ and $Q_{66}$ are reduced. For the research at hand, all terms to be reduced will be conservatively set equal to zero.

The redistribution schemes are basically the same as those presented for an interactive scheme except greater care must be given to defining what loads must be redistributed and what loads may be accepted by a neighboring layer. A complete discussion is given in Appendix B.

\subsection{Results and Example Calculations for GR/EP Laminates}

In this section, example calculations for various laminates will be presented using the simulation techniques developed in the previous sections.

As a first example, a [0/土15]. GR/EP laminate under uniaxial loading is examined using an interactive failure criterion. One thousand failure loads were simulated for each redistribution scheme, and identical results were achieved for each scheme. The distribution function was approximated using a median ranking (see Appendix A). The reliability versus load results are presented in Figure(6). Figure(6) also contains reliability limit information calculated using the method of Section(3). It is seen that these limits do indeed bound the predicted reliability values.

For this particular case, the choice of redistribution scheme used in the simulation had no effect on the calculated reliability. Though this result is 
somewhat unexpected, one explanation is that this is due to the small relative orientation between the layers. The laminate can attain a rather large load before the $\pm 15^{\circ}$ layers begin failing. These layers should have approximately the same strengths; thus it is likely that regardless of which layer fails first, the +15 or -15 , the other layer will also fail upon redistribution. This occurrence would leave the $0^{\circ}$ layer carrying the entire load, which it could not sustain, and lead to failure of the laminate.

Figure(7) displays the results for a noninteractive analysis of the same situaution. With a modal failure criterion, the choice of redistribution scheme had a small but noticeable effect. A local, orientation sensitive redistribution predicted the highest probability of failure for a given load and a global redistribution predicted the lowest, though the difference was generally small. The projected limits given in Section(3) properly bound the predicted reliability.

A similar analysis is presented in Figures(8) and (9), for a $[90 / \pm 45 / 0]$ 。 GR/EP laminate. The results using an interactive failure criterion are shown in Figures(8). The effect of of the various redistribution methods is much more pronounced for this case in the high reliability range $(\approx R>0.80)$. The highest reliability at a given load is predicted by a global redistribution, followed by tapered orientation insensitive, local orientation insensitive, tapered orientation sensitive and finally local orientation sensitive. Once again, the limits bound the predicted reliability values.

In Figure(9) the results using a noninteractive failure criterion are given. The large difference between the upper and lower bound limits is due to the large difference in lamina orientations.

\section{Summary and Conclusions}

This paper has presented two methods, non-interactive(PIA) and interactive(MDE), by which the reliability of a single continuous fiber composite lamina may be analysed. At this time insufficient experimental data exists to give any preference to one over the other. The concept of a reliability surface for the lamina in stress space was also presented, and a mapping technique was introduced to transform the surfaces to a non-dimensionalized space where they take on a spherical shape and become material independent.

The individual lamina reliabilities were then combined to produce formal upper and lower bound limits of reliability for a composite laminate under multiaxial loading. These limits, which do not account for free-edge 


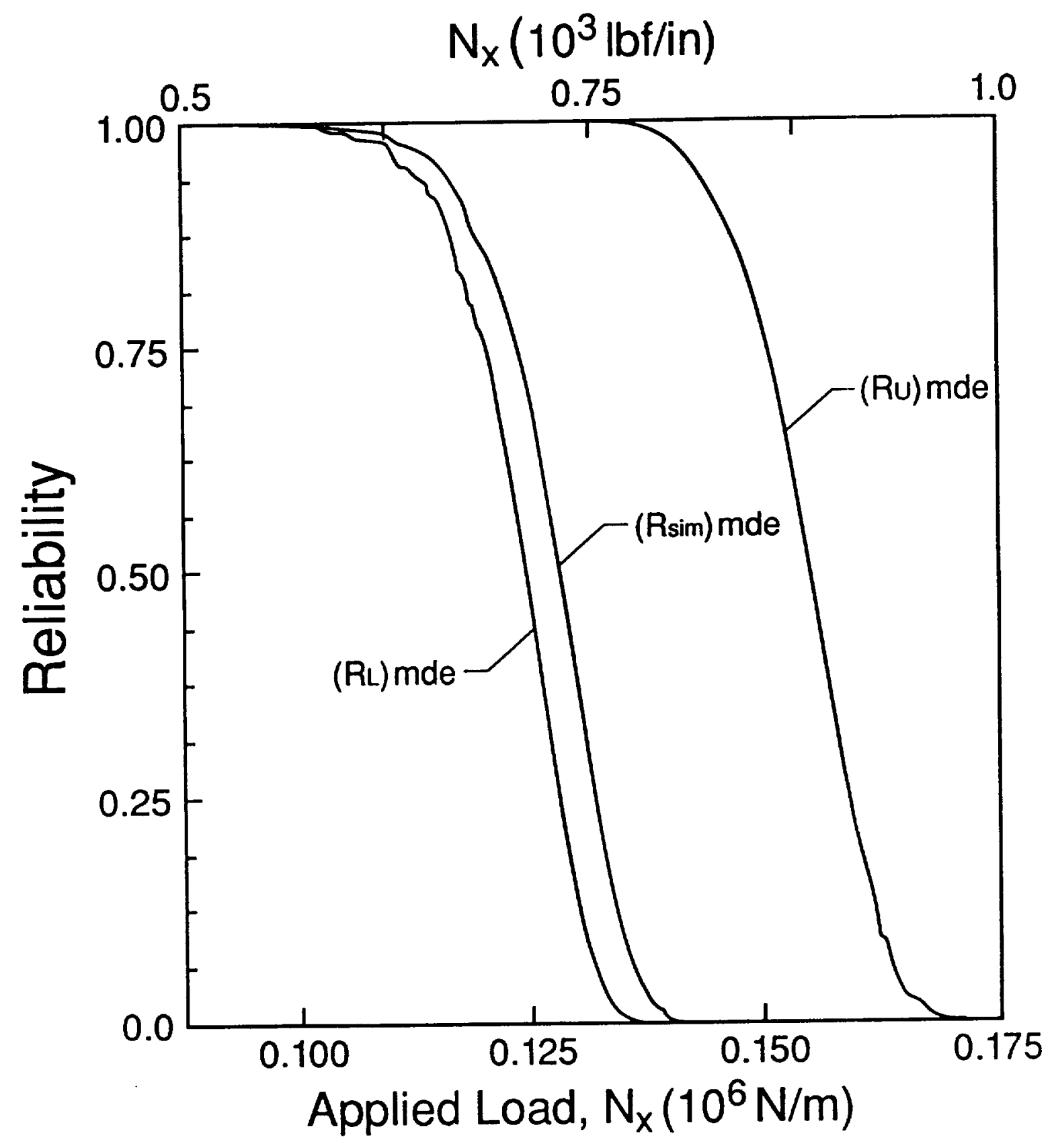

Figure 6: Interactive reliability as a function of the applied load for a $[0 / \pm 15]$, GR/EP laminate 


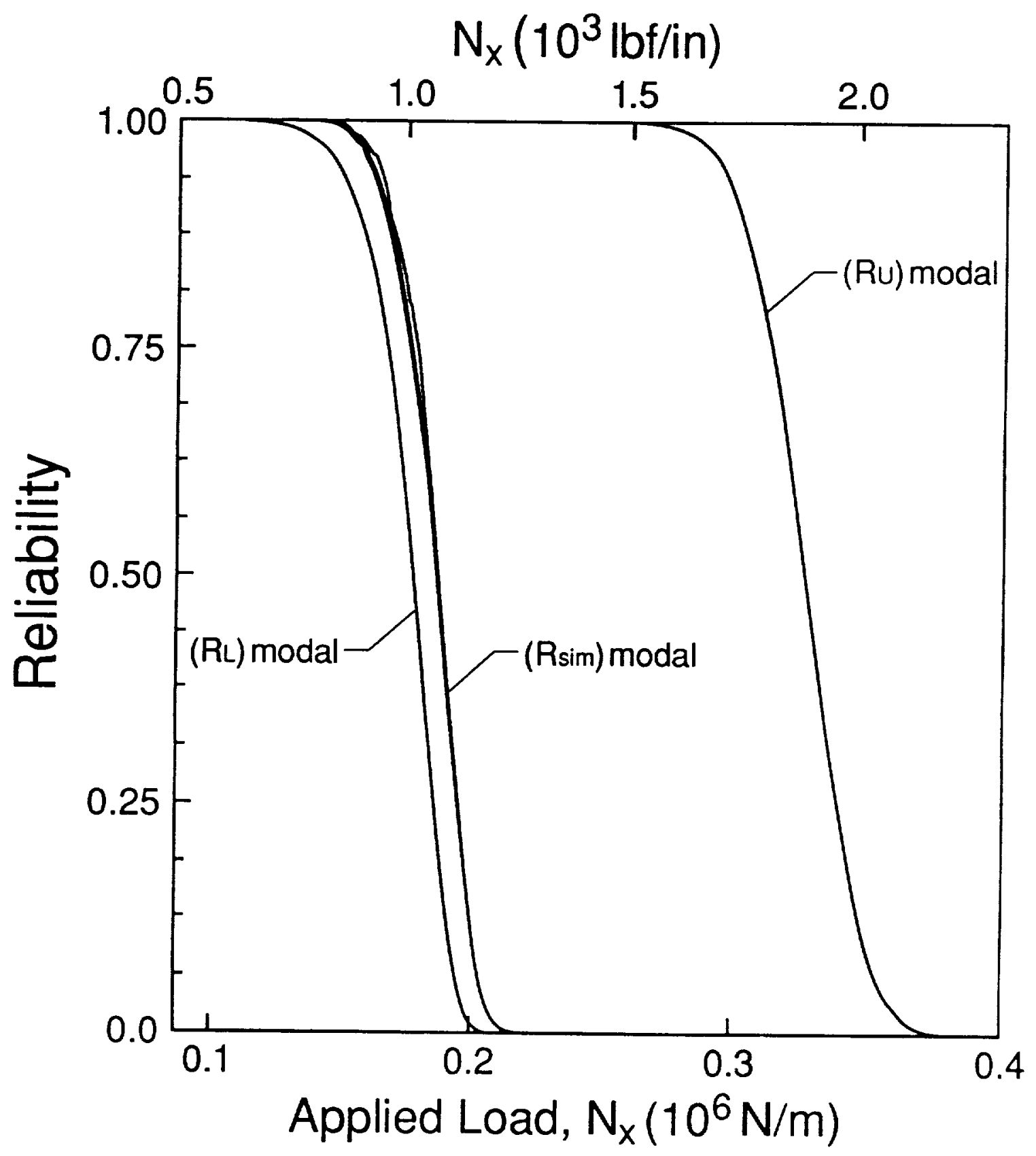

Figure 7 : Noninteractive reliability as a function of the applied load for a $[0 / \pm 15]_{s}$ GR/EP laminate 


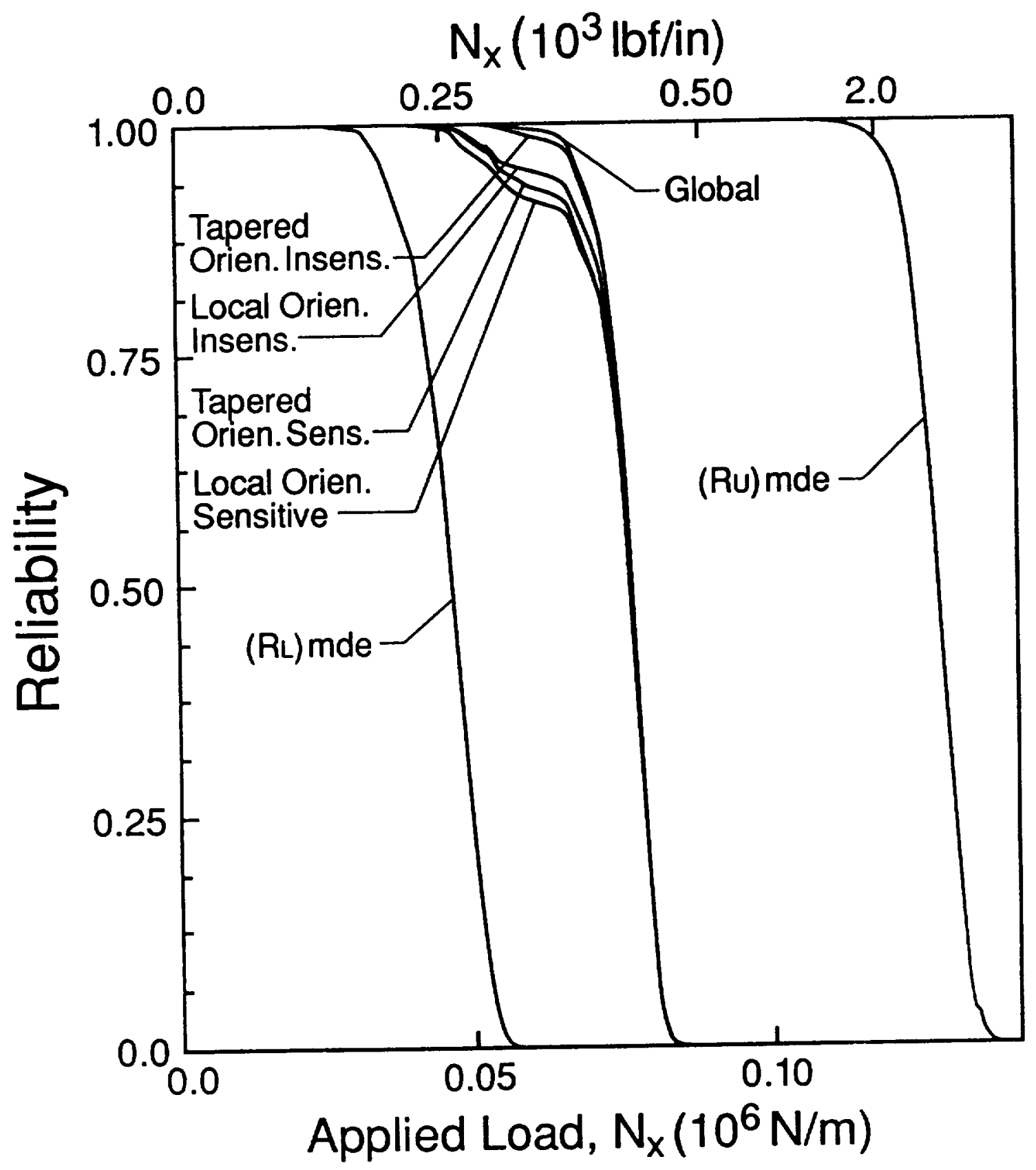

Figure 8: Interactive reliability as a function of the applied load for a $[90 /=45 / 0]$, GR/EP laminate 


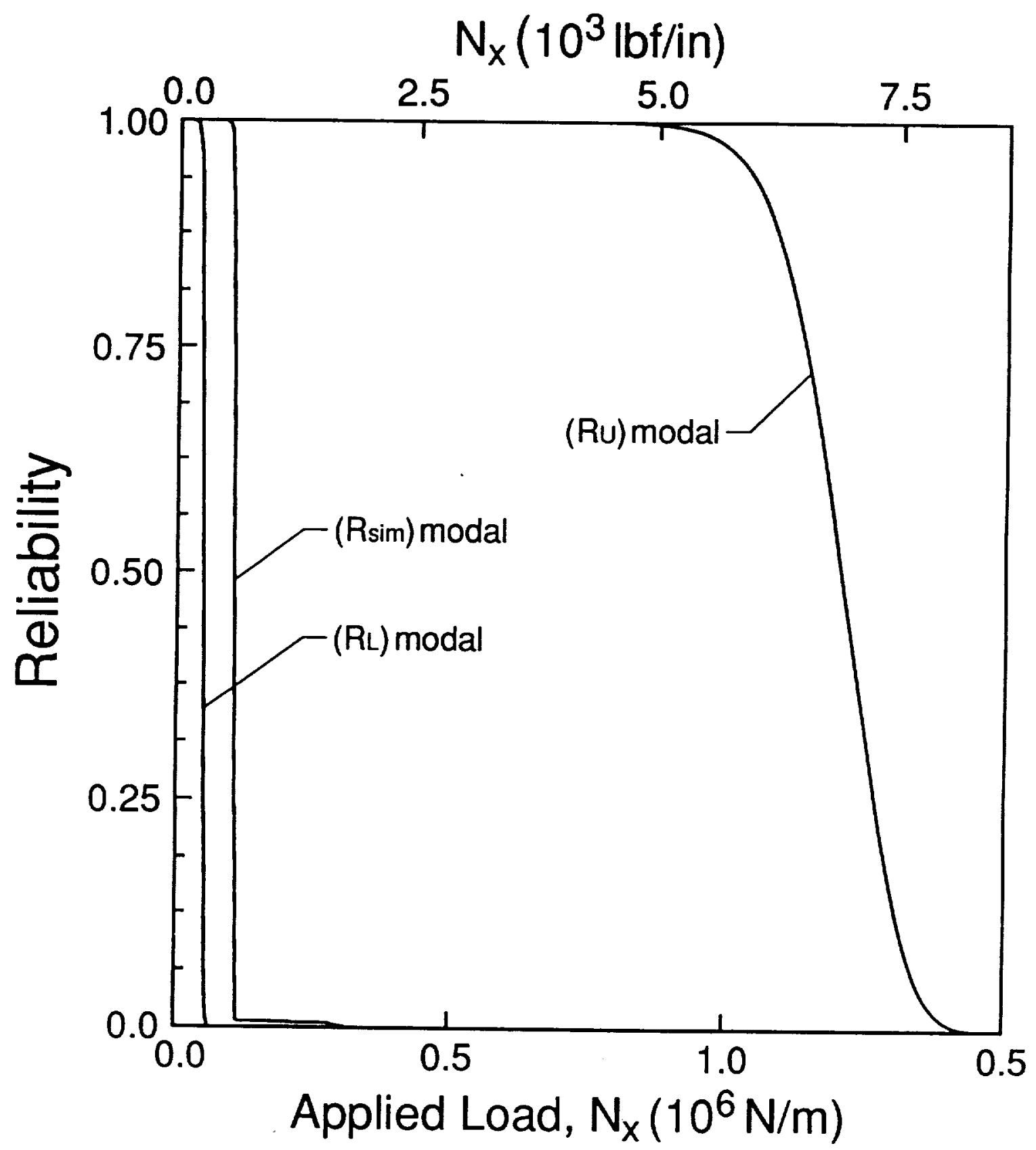

Figure 9: Noninteractive reliability as a function of the applied load for a $[90 / \pm 45 / 0]_{s}$ GR/EP laminate 
induced delamination effects, were derived for both interactive and modal failure considerations. Surfaces of constant reliability in a load space were illustrated for an example laminate. Further example cases demonstrated the bounds over a range of loads. These calculations were found to give consistent results. The proposed load redistribution schemes were implemented to more closely define the reliability state; the degree of dependence of the reliability on the particular load redistribution scheme depends on the laminate stacking sequence. Present research is under way to more accurately model the failure modes and load redistributions.

\section{Acknowledgements}

This research was supported by the NASA-Lewis Research Center under Contract NAG3862.

\section{References}

[1] Pipes, R. Byron and Pagano, N.J., "Interlaminar Stresses in Composite Laminates Under Uniform Axial Extension," J. of Composite Materials, 1970, pp.538-548.

[2] Pagano, N.J. and Pipes, R. Byron, "The Influence of Stacking Sequence on Laminate Strength," J. of Composite Materials, 1971, pp.50-57.

[3] Wetherhold, R.C., "Statistical Distribution of Strength of FiberReinforced Composite Materials," Polymer Composites 7 (1986), pp.116-123.

[4] Mann, N.R., Schafer, R.E. and Singpurwalla, N.D., "Methods for Statistical Analysis Analysis or Reliability and Life Data," John Wiley and Sons, New York, (1974).

[5] Wetherhold, R.C., "Statistics of Fracture of Composite Materials Under Multiaxial Loading," Ph.D. Dissertation, University of Delaware, (1983). 
[6] Sun, C.T. and Yamada, S.E., "Strength Distribution of a Unidirectional Fiber Composite," J. Comp. Matls. 12 (1978), pp. 169-176.

[7] Yamada, S.E. and Sun, C.T., "Analysis of Laminate Strength and Its Distribution," J. of Comp. Matls. 12 (1978), pp.275-284.

[8] Duffy, Steven F. and Manderscheid, Jane M., "Noninteractive Macroscopic Reliability Model for Ceramic Matrix Composites With Orthotropic Material Symmetry," 34th International Gas Turbine and Aeroengine Congress and Exposition sponsored by A.S.M.E., Toronto, Canada, 1989.

[9] Wetherhold, Robert C. and Pipes, R. Byron, "Statistics of Fracture of Composite Materials Under Multiaxial Loading," Materials Science and Engineering 68 (1984), pp.113-118.

[10] Cassenti, B.N., "Probabilistic Static Failure of Composite Material," AIA A Journal 22 (1984), pp.103-110.

[11] De Roo, P. and Paluch, B., "Application of a Multiaxial Probabilistic Failure Criterion to a Unidirectional Composite," Developments in the Science and Technology of Composite Materials; Proc. of the First European Conference on Composite Materials and Exhibition, Association Europeene des Materiaux Composites,France, 1985, pp.328-334.

[12] Johnson, L.G., "The Median Ranks of Sample Values in Their Population With an Application to Certain Fatigue Studies," Industrial Math, vol. 2,1971

[13] Yang, Ling, "Reliability of Composite Laminates," Mech. Struct. \& Mach., 16 (1988-89), pp.523-536.

[14] Fukunaga, Hisao; Chou, Tsu-Wei and Fukuda, Hiroshi, "Probabilistic Strength Analyses of Interlaminated Hybrid Composites," Composites Science and Technology, 35 (1989), pp.331-345. 


\section{A Laminate Reliability Analysis With Interac- tive Failure Criterion and Load Redistribution Considerations}

\section{Part One: Predicting the Failure Load}

It is desired to have the simulation emulate a load-controlled test with ramp loading. Thus the applied load must increase from zero to some specified magnitude along a line of constant loading ratios(i.e. $N_{x}: N_{y}$ $=$ constant, $N_{x}: N_{x y}=$ constant). In order to predict the first failure load a nominal loading must initially be applied and the resulting stress state calculated. This initial loading is derived from the applied load in a way such that the ratios of all the corresponding loading elements remain unchanged, but the value of the initial loading element in the maximum loading direction is set equal to unity. For example, for a specified applied load of $\underline{N}=\left[\begin{array}{lll}400 . & 100 . & 0 .\end{array}\right]^{T}$ the reduced initial load would be $\underline{N}_{\text {initial }}=\left[\begin{array}{lll}1.00 & 0.25 & 0.00\end{array}\right]^{T}$.

At this loading, the stress state in each layer may be determined through application of the constitutive relationship. With the stress states known the f-values may be calculated for each layer using Equation(6). From these values, the loading at which the first failure occurs may be predicted using the following expression.

$$
\underline{N}_{\text {new }}=\frac{1}{\sqrt{\left[\left(f_{\text {old }}\right)_{i}\right]_{\text {max }}}} N_{\text {old }}
$$

where $i$ corresponds to the various layers.

At failure, the load is redistributed. If further failures occur, the loads corresponding to the newly failed layers must also be redistributed and the layers now remaining checked for further failures. If no additional failures have occurred, the process just described in this section may be used to predict the next failure load.

Part Two: Redistributing the Load of a Failed Ply

The f-value of each layer is computed, and if failure has occurred its stiffness matrix is set equal to zero and its loading redistributed locally in the following general manner for all of the redistribution schemes outlined in Section 4.1.

Step 1. Moving to the appropriate neighboring layer: If that layer has 
either failed or does not exist(i.e. the layer that has failed is an outside layer thus having no neighbor on the one side), its portion of the redistribution load is stored for global redistribution. If however that layer has not failed, this load is stored in a buffer of local stress effects corresponding to the layer(i.e. the neighboring layer).

Having carried out step(1) for each layer, the entire loading has now been characterized and stored into three groups: original stresses still carried by the surviving layers, local stress effect buffers(one corresponding to each layer) and a global buffer. Here it is assumed that the original stresses and the global stresses are distributed according to the laminate constitutive law, and that the local stresses are distributed locally and do not obey the deformation assumptions. This seems is reasonable in that localized stresses should cause localized displacements. Proceeding on,

Step 2. The portion of the applied load conforming to constitutive theory, which will be defined here to be called $N^{\prime}$, is given by summing the stresses carried by the surviving layers and the global stresses and multiplying by the layer thickness.

$$
\left\{\begin{array}{c}
N_{x}^{\prime} \\
N_{y}^{\prime} \\
N_{x y}{ }^{\prime}
\end{array}\right\}=t \times\left[\sum_{i=1}^{n}\left\{\begin{array}{c}
\sigma_{x_{C A R A I E D}} \\
\sigma_{y_{C A R R I E D}} \\
\tau_{x y_{C A R R I E D}}
\end{array}\right\}_{i}+\left\{\begin{array}{c}
\text { Global }_{x} \\
\text { Global }_{y} \\
\text { Global }_{x y}
\end{array}\right\}\right]
$$

Step 3. Via laminate constitutive theory, the stress state, $\underline{\sigma}^{\prime}$, is calculated in geometric coordinates using the re-evaluated stiffness matrices(which will take into account any failed plies) and the primed load calculated in $\operatorname{step}(2)$.

$$
\left\{\begin{array}{c}
\sigma_{x}{ }^{\prime} \\
\sigma_{y}{ }^{\prime} \\
\tau_{x y}{ }^{\prime}
\end{array}\right\}_{i}=[\bar{Q}]_{i}[A]^{-1}\left\{\begin{array}{c}
N_{x}{ }^{\prime} \\
N_{y}{ }^{\prime} \\
N_{x y}{ }^{\prime}
\end{array}\right\}
$$

Step 4. The redistributed stress state in each layer is then calculated by adding the local effects directly to the primed stresses determined in $\operatorname{step}(3)$.

$$
\left\{\begin{array}{c}
\sigma_{x} \\
\sigma_{y} \\
\tau_{x y}
\end{array}\right\}_{i}=\left\{\begin{array}{c}
\sigma_{x}^{\prime} \\
\sigma_{y}^{\prime} \\
\tau_{x y}{ }^{\prime}
\end{array}\right\}_{i}+\left\{\begin{array}{c}
\sigma_{x_{L O C A L}} \\
\sigma_{y_{L O C A L}} \\
\tau_{x y_{L O C A L}}
\end{array}\right\}_{i}
$$

The f-values are recalculated under the newly redistributed stress state. In the event that new failures have occurred, the redistribution scheme is repeated. If however, no new failures occur, and there are surviving plies 
remaining, the load is incremented to the next predicted failure load using the method presented in Part One.

Part Three: Reliability Determination

The reliability of the laminate under the applied load is determined using the results of a Monte Carlo analysis. An $n$ sized sample space of loads corresponding to complete laminate failure are simulated using the methods of Parts One and Two. This data may be ranked using a median ranking[12] to approximate the probability of failure distribution function,

$$
p_{j}=\frac{j-0.3}{n+0.4} \quad j=1,2, \ldots, n
$$

The value of the distribution function at the applied load may be determined through a linear interpolation, and subtracting this value from unity yields the laminate reliability for the specified load.

$$
R=1-p_{j} \underline{N}_{A P P L 1 E D}
$$

The value of $n$ should be chosen such that there are a reasonable number of both successes and failures $(\sim 20$ minimum of each $)$.

\section{B Laminate Reliability Analysis With a Non-interactive Modal Failure Criterion and Load Redistribu- tion Considerations}

Part One: Predicting the Failure Load

As discussed in Part One of Appendix A, the simulation should portray a load-controlled test to failure. To this end, an initial loading relatively close to zero is derived from the specified applied loading as demonstrated in Appendix A. Using the constitutive law, the stress state in each layer can be determined under the initial loading. This information can then be used with the failure functions of Equations(21-23) to compute the f-values for individual modes of each layer.

Potentially, there are three possible failure modes(longitudinal, transverse and shear) in each layer. However, depending on the type of loading and the ply orientations, all of these modes may not be active. That is, some lamina may not be stressed in a particular mode either during initial loading or after partial failure of the laminate. The number of active modes is equal 
to the number of non-zero f-values calculated under the initial loading. This leads to the following definition of laminate failure:

Final laminate failure has occurred when all active failure modes have failed

The load corresponding to the first modal failure can be predicted with the following expression.

$$
\underline{N}_{\text {new }}=\frac{1}{\left[\left(f_{j}\right)_{i}\right]_{\max }} N_{\text {old }}
$$

At failure, the load is redistributed. If no further failures have occurred, the same process is used to predict the next failure load.

Part Two: Redistributing the Load of a Failed Mode

If one or more modal failures have occurred in a layer, the following general procedure is followed for any of the local load redistribution schemes discussed in Section 4.1.

Step 1. Within the layer containing the modal failure, the loads to be maintained, or carried, and those to be redistributed are defined. Both of these loading vectors are transformed from material to geometric coordinates.

Step 2. Moving to the appropriate neighboring layer: If there is no neighboring layer, its portion of the distribution vector is stored for global distribution. Otherwise, the distribution vector is transformed to the material coordinates of the neighboring ply. The stress terms corresponding to the survivng modes(if any) of the neighboring ply are stored in a buffer of local stress effects for that layer, and the stress terms corresponding to the failed modes(if any) are stored in a global buffer. The elements of both buffers are transformed back to the geometric coordinate system and stored.

After performing steps(1) and (2) for all layers, the total loading vector has been divided into three groups: the stresses still carried by the survivng modes, local stress effect buffers and a global stress buffer. As previously assumed, the stresses carried by the surviving modes and the global stresses are taken to obey laminate constitutive laws, and the local stress effects do not. Continuing with the redistribution procedure:

Step 3. Same as Step 2 in Appendix A.

Step 4. Same as Step 3 in Appendix A.

Step 5. Same as Step 4 in Appendix A.

There are two criterions by which complete laminate failure is defined. 
the first was given in Part One to be when all active modes have failed. The second is when the $[A]$ matrix, which represents the in-plane stiffness response, becomes singular. This happens when the only unfailed modes remaining in the layers are the shear modes. In this case the resulting stiffness matrices become,

$$
[Q]_{i}=\left[\begin{array}{ccc}
0 & 0 & 0 \\
0 & 0 & 0 \\
0 & 0 & Q_{66}
\end{array}\right]
$$

The occurrence of this event is analogous to reaching the yield stress in a linear elastic-perfectly plastic material; the application of any additional load causes an indeterminantly large deflection.

Part Three: Reliability Determination

See Part Three of Appendix A. 


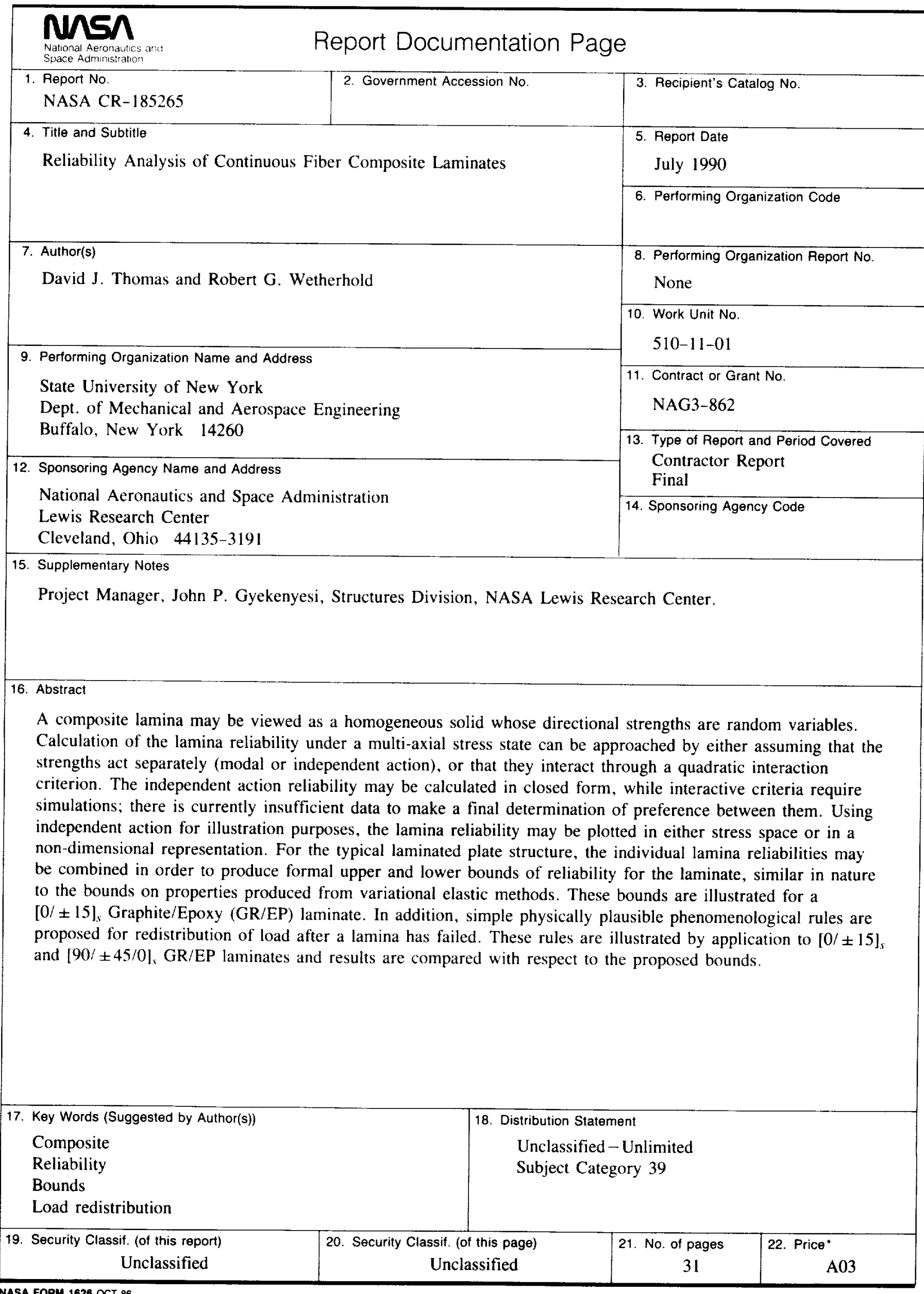

